\title{
Parity violation correlations in light muonic atoms
}

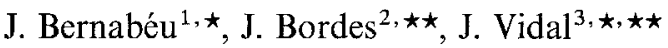 \\ 1 Department de Física Teòrica, Universitat de València, c/Dr Moliner, 50, E-46100 Burjassot-València, Spain \\ 2 Rutherford Appleton Laboratory, Chilton, Didcot, Oxon OX11 OQX, UK \\ 3 Randall Physics Laboratory, University of Michigan, Ann Arbor, MI 48109, USA
}

Received 27 July 1988

\begin{abstract}
The $2 S-1 S$ transition in light muonic atoms is very sensitive to parity violation correlations induced via neutral currents. Observables depending on these transitions such as the photon polarization and the angular correlation between the emitted radiation and the atom polarization are a clear signal of weak neutral currents in atoms. We find the relation between the lepton and quark couplings and these observables emphasizing the effect of the nuclear spin. The results expected in muonic atoms $\mu-{ }^{4} \mathrm{He}$ and $\mu-{ }^{3} \mathrm{He}$ are given.
\end{abstract}

\section{Introduction}

The study of neutral currents in atoms has been the subject of great interest for many years [1]. In spite of the already abundant data related to neutral current effects from high energy physics [2], the fact that very different physics is involved has continued the interest of the weak neutral currents in atoms: they provide a framework in which quarks act coherently with regard to the weak interactions and the momentum transfer relevant is of the order of several $\mathrm{keV}$ to $\mathrm{MeV}$ compared to the scale of $\mathrm{GeV}$ in high energy physics experiments. Both class of experiments are then complementary allowing the comparison of the parameters of the theory at such different energies.

Heavy atoms have been from the very beginning the favourite atomic system for the experimental study of these processes [3]. Furthermore, at present they constitute the only uncontested agreement among measurements performed by different groups. The agreement with the theoretical predictions within the framework of the standard model is also remarkable.

\footnotetext{
* Also at IFIC, Centre Mixte Universitat de València-CSIC, Burjassot, València

$\star \star$ On leave from Department de Física Teòrica, Universitat de
} València
There is, however, the drawback that in general they only provide the determination of a definite combination of coupling constants between lepton and quarks, namely $3 \tilde{\gamma}(Z+N)+\tilde{\alpha}(Z-N)$ (where $Z$ and $N$ are the number of protons and neutrons inside the nucleus and $\tilde{\alpha}, \tilde{\gamma}$ are the scalar coupling constants in the Hung-Sakurai parametrization [6]) while, at present they cannot reach information about $\widetilde{\beta}$ and $\widetilde{\delta}$ since they appear in the contribution depending on the nuclear spin and hence on the nucleons outside closed shells.

There are at present a considerable number of experiments under way to measure parity violation correlations in light atoms [4]. Measurements in hydrogenic atoms such as hydrogen, deuterium, tritium and helium, are particularly important because such experiments will make it possible to determine both the nucleus-spin dependent and the nucleus-spin independent coupling constants. Such measurements will be able to provide a more accurate determination of the Weinberg angle and, occasionally, to study intermediate massive neutral bosons in theories beyond the standard model [5]. Finally, from the theoretical point of view, these results will offer a much easier interpretation because of the simplicity of these systems.

In this paper we want to discuss the attractive features that offer light muonic atoms to study parity violation effects. We will focus our attention on the dipole magnetic transition between the mixing of the near degeneracy $2 S-2 P$ states and the $1 S$ state [7]. We will emphasize the role of the nuclear spin, finding explicitly the dependence of observables on the coupling constants $\widetilde{\beta}$ and $\widetilde{\delta}$ that cannot be reached with a scalar nucleus or are far from the experimental precision in the experiments under way with heavy atoms.

The paper is organized as follows: in Sect. 2 we present the virtues of muonic atoms in searching for parity violation effects. We discuss in particular the 
case of light muonic atoms. The appropriate formalism and the observables based on the measurement of the dipole magnetic transition are given in Sect. 3. Section 4 is devoted to establish the relation between the parameters of the standard electroweak theory and the observables described in Sect. 3. Finally, the results for the observables in $\mu-{ }^{3} \mathrm{He}$ and $\mu-{ }^{4} \mathrm{He}$ are given in Sect. 5 as well as the conclusions.

\section{Muonic atoms. Parity violation correlations}

The interaction between lepton and nucleus can be described by the effective Hamiltonian:

$H=H_{0}+H_{W}$

where $H_{0}\left(H_{W}\right)$ is the electromagnetic(weak) Hamiltonian. $H_{W}$ can be split into two pieces:

$H_{W}=H_{W}(\mathrm{PC})+H_{W}(\mathrm{PV})$

the parity conserving piece, $H_{W}(\mathrm{PC})$, will be ignored when compared to $H_{0}$ since it is parity conserving too.

On the other hand, as a consequence of the parity violating piece, $H_{W}(\mathrm{PV})$, the eigenstates of the complete Hamiltonian have a non-definite parity. At the dominant order in the weak Hamiltonian, the parity mixing is given by the interference between the electromagnetic Hamiltonian and the parity violating piece of the weak Hamiltonian. Therefore, the eigenstates of $H$ in (2.1) are mixing between parity defined states of the electromagnetic Hamiltonian $H_{0}$.

Thus, an indication of the weak neutral currents in atoms is the measurement of the nonvanishing expectation value of a pseudoscalar observable. These observables have a typical structure such as:

$O=\eta \frac{T_{1}}{T_{0}}$

written in terms of the electromagnetic transition amplitudes of the admixed states. In the former equation $T_{1}\left(T_{0}\right)$ is the parity allowed (forbidden) transition amplitude of the admixed states and $\eta$ is the mixing parameter. Using time independent perturbation theory, $\eta$ is given by:

$\eta=\frac{\left\langle H_{W}\right\rangle}{\Delta E}$

where $\left\langle H_{W}\right\rangle$ is the expectation value of $H_{W}(\mathrm{PV})$ between the opposite parity states and $\Delta E$ its difference of energy.

Equations $(2.3,2.4)$ give the key to the selection of the electromagnetic transitions sensible to the neutral current effects. Focussing our attention to light atoms, the signal $O$ will depend on the following factors:

i) The mixing parameter $\eta$ depends on two factors: the energy difference and the overlapping of the leptonic and nuclear wave functions.

ii) The enhancement factor $T_{1} / T_{0}$ depending on the parity forbidden electromagnetic amplitude $T_{0}$.
In heavy atoms on the other hand, the enhancement is provided by the " $Z$ ' factor", coming from the coherent interaction of the lepton with the quarks in the nucleus [1].

As a first observation, the ratio between the electromagnetic amplitudes as well as the difference of energy between the admixed states, are independent of the nature of the lepton. However, the overlapping of the leptons and nuclear probabilities does depend on the lepton. The fact that the weak interaction is short ranged determines that the more the probability distributions of the leptons lay inside the nucleus, the bigger the overlapping factor is. From this point of view muonic atoms give a larger signal factor than the electronic ones. Considering the Bohr radius in both class of atoms, its ratio is given by the relation of lepton masses, namely $a_{\mu} / a_{e} \sim \frac{1}{205}$, hence the expectation value of $H_{W}$ is enlarged by a large factor if one considers muonic atoms. This fact was first considered in [7] and recently has been the subject of renewed experimental interest [9].

Let us now consider in more detail the case of muonic atoms. Since the muon Bohr radius is (already for $n \sim 14$ ) inside the electronic orbits, one can study these atoms as a hydrogen-type one. These systems are very sensitive to parity mixing states with equal quantum number ( $n \geqq 2$ ). In fact, in the Bohr atom the parity mixing between states is complete, being themselves degenerate, they are however split through vacuum polarization and finite size effects. Therefore they are slightly separated as compared to the spacing of states with different $n$ quantum number. On top of that, additional enhancement, as it was argued in $i$ ) above, can be obtained if the main contributions to the hyperfine splitting, namely the finite size effect of the nucleus and vacuum polarization, have opposite sign. We find this phenomenon in the low energy levels of light muonic atoms, making them a suitable system to study observables of the type described in (2.3).

In particular, the $2 S-1 S$ transition in light muonic atoms is very sensitive to parity mixing, since the $2 S$ state is strongly mixed with the opposite parity state $2 P$.

Another factor plays a crucial role in the construction of observables from the $2 S-1 S$ transition. When the muon is captured by the atoms, it begins the electromagnetic cascade to the fundamental level. All excepted the $2 S$ state, decay through radiative electric dipole transition $(E 1)$. Any parity violating effect in these transitions will depend, apart from the mixing parameter $\eta$, on the non-allowed transition, therefore this effect will be in general very small. In the $2 S$ level, on the contrary, the transition takes place through the radiation of two electric dipole photons or a magnetic dipole one $(M 1)$. The last one is hindered due to the change of the radial quantum number, in fact is only nonzero through relativistic effects and retardation. When the $2 S-2 P$ mixing is considered (they are nearly degenerated), the electromagnetic transition acquires 
an $E 1$ component from the $2 P-1 S$ transition. Thus the interest of this kind of transition from the point of view of the enhancement factor ii) discussed above.

Before finishing this section let us comment on the experimental feasibility of measuring parity violating effects in these transitions. They will rely on the measure of the magnetic dipole transition $(M 1)$ as commented before, this is extremely difficult because of the low $M 1$ branching ratio of the weakly populated $2 S$ level, however the development of the cyclotrontrap by Simons [10] restored the interest in these kind of observables since high stopping densities for muons under the suitable conditions can be obtained.

\section{The magnetic dipole transition in light muonic atoms. Observables}

In this section we aim to develop the formalism suitable to the construction of the observables of the form given in (2.3). We will restrict to the discussion of two specific ones, relying on the measurement of the magnetic dipole transition, namely the photon polarization and the asymmetry in the emission of photons from the electromagnetic transitions. A complete discussion of the observables can be found in [11]. As we argued before, we will focus our attention on the $2 S-1 S$ transition in light muonic atoms. We consider here the case in which the spin of the nucleus is $\frac{1}{2}$. The result for zero-spin nucleus can be obtained as a by product of the results presented here.

In general, for a nucleus of spin $I$, the hyperfine states of the atom are given by:

$\left|F M ; n L_{J} ; I\right\rangle=\sum_{m_{I} m_{J}}\left(J I F \mid m_{J} m_{I} M\right)\left|n L_{J} m_{J}\right\rangle\left|I m_{I}\right\rangle$

where $\left|n L_{J} m_{J}\right\rangle$ is the muonic wave function that, in a Schrödinger type representation is given by the solution of the appropriate relativistic wave equation with the electromagnetic Hamiltonian $H_{0}[12],\left|I m_{I}\right\rangle$ is the nuclear state we do not need to specify here apart from the spin content. The pair $F M$ represents the total and third component of the angular momentum of the hyperfine atomic state, and from the Clebsh-Gordon coefficient one has $F=|J-I| \cdots$ $|J+I|$ and $M=m_{J}+m_{I}$. The $2 S-2 P$ admixture is given by the eigenstates of the Hamiltonian (2.1) (only the admixture among states with the same $n$ is significant, then we only consider the admixture with $2 P$ states). In the particular case $I=\frac{1}{2}$, the physical states read:

$$
\begin{aligned}
|F M\rangle= & \left|F M ; 2 S_{1 / 2} ; \frac{1}{2}\right\rangle \\
& +\sum_{J=1 / 2,3 / 2} \eta(F, J)\left|F M ; 2 P_{J} ; \frac{1}{2}\right\rangle .
\end{aligned}
$$

Since the total angular momentum is not affected through the weak interaction, the mixing parameter is dependent on $F$ and $J$. Hence $\eta(F, J)$. Also it is obtained, using perturbation theory, from the weak Hamiltonian $H_{W}(\mathrm{PV})$.
We are now in a position of writing the radiative transition between two hyperfine states of the $2 S$ and $1 S$ levels. It will be the dipole magnetic transition $M 1$, from the $2 S_{1 / 2}-1 S_{1 / 2}$ transition with an $E 1$ component from the $2 P-1 S_{1 / 2}$ transition, the mixing given by the parameter $\eta$ (there is no mixing in the $1 S$ energy level).

In an obvious notation, the complete amplitude is:

$$
\begin{gathered}
A\left(F M \rightarrow F^{\prime} M^{\prime}\right)=A^{M 1}\left(F M ; 2 S_{1 / 2} \rightarrow F^{\prime} M^{\prime} ; 1 S_{1 / 2}\right) \\
\quad\left\{1+\eta\left(F, \frac{1}{2}\right) \frac{A^{E 1}\left(F M ; 2 P_{1 / 2} \rightarrow F^{\prime} M^{\prime} ; 1 S_{1 / 2}\right)}{A^{M 1}\left(F M ; 2 S_{1 / 2} \rightarrow F^{\prime} M^{\prime} ; 1 S_{1 / 2}\right)}\right. \\
\left.+\eta\left(F, \frac{3}{2}\right) \frac{A^{E 1}\left(F M ; 2 P_{3 / 2} \rightarrow F^{\prime} M^{\prime} ; 1 S_{1 / 2}\right)}{A^{M 1}\left(F M ; 2 S_{1 / 2} \rightarrow F^{\prime} M^{\prime} ; 1 S_{1 / 2}\right)}\right\}
\end{gathered}
$$

where the electromagnetic amplitudes are given by

$$
\begin{gathered}
A^{M 1}\left(F M \rightarrow F^{\prime} M^{\prime}\right)=\sqrt{\frac{2 \pi \alpha}{\Delta E_{s}} \frac{(\alpha Z)^{4}}{3^{3}}} \\
\sum_{\lambda= \pm 1}\left(\frac{1}{2} \frac{1}{2} F \mid \frac{\lambda}{2} M-\frac{\lambda}{2}\right)\left(\frac{1}{2} \frac{1}{2} F^{\prime} \mid-\frac{\lambda}{2} M-\frac{\lambda}{2}\right) \delta_{M M^{\prime}+\lambda} \varepsilon_{\lambda} \\
A^{E 1}\left(F M \rightarrow F^{\prime} M^{\prime}\right)=-i \sqrt{\frac{2 \pi \alpha}{\Delta E_{p}}}(\alpha Z) \frac{2^{5}}{3^{4} \sqrt{3}} \\
\sum_{\lambda= \pm 1}\left(\frac{1}{2} \frac{1}{2} F \mid \frac{\lambda}{2} M-\frac{\lambda}{2}\right)\left(\frac{1}{2} \frac{1}{2} F^{\prime} \mid-\frac{\lambda}{2} M-\frac{\lambda}{2}\right) \delta_{M M^{\prime}+\lambda} \varepsilon_{\lambda}
\end{gathered}
$$

with $\Delta E_{s}\left(\Delta E_{p}\right)$ the energy difference between the $2 S-1 S(2 P-1 S)$ hyperfine states involved and $\varepsilon_{+}$stands for the polarization of the emitted photon in the transition.

From (3.4) one finds the enhancement factor related to the amplitude that we discussed in Sect. 2 since the ratio between them is $\sim(\alpha Z)^{-3}$.

Any parity violation observable with the structure given in (2.3) will depend on the difference between the transition probabilities for the emission of $\varepsilon_{+}$and $\varepsilon_{-}$polarized photons. Thus, we can define the photon polarization:

$a\left(F, F^{\prime}\right)=\frac{W_{+}\left(F, F^{\prime}\right)-W_{-}\left(F, F^{\prime}\right)}{W_{+}\left(F, F^{\prime}\right)+W_{-}\left(F, F^{\prime}\right)}$

where $W_{\varepsilon}\left(F, F^{\prime}\right)$ are the above mentioned probabilities. Ignoring the hyperfine splitting as compared to the separation between the $2 S-1 S$ levels, one easily finds:

$$
\begin{aligned}
a\left(F, F^{\prime}\right)= & \frac{-64}{3 \sqrt{3}(\alpha Z)^{3}} \\
& \cdot\left\{\operatorname{Im} \eta\left(F, \frac{1}{2}\right)+\frac{1}{8} \operatorname{Im} \eta\left(F, \frac{3}{2}\right) \delta_{F, 1}\right\} .
\end{aligned}
$$

One should expect this dependence on the mixing parameter if time reversal invariance is assumed in the weak interactions since the amplitudes $A^{E 1}$ and $A^{M 1}$ are relatively imaginary.

From the difficulty of measuring the polarization of the magnetic radiation, a more convenient observable is given by the detection of the angular correlation between the emitted photon and the initial polariz- 
ation of the atom. We briefly describe this observable and find its relation to the mixing parameter.

The initial state of the atom in the state of spin $F$ is described by the density matrix:

$\rho^{F}=\frac{1}{2 F+1}\left\{1+\frac{3}{F+1} \mathbf{P} \cdot \mathbf{F}\right\}$

where $\mathbf{P}$ is the polarization of the atom. Tensorial polarizations have been omitted since they are even under parity hence they do not contribute to the observables we are discussing here.

The angular distribution is then:

$I^{\left(F, F^{\prime}\right.}(\theta)=\frac{\left(\Delta E_{F, F^{\prime}}\right)}{(2 \pi)^{2}} \operatorname{Tr}\left(A \rho^{F} A^{+}\right)$

being $A$ a shorthand notation for the amplitude in (3.3) and $\Delta E_{F, F^{\prime}}$ the energy difference between the initial and final hyperfine states.

Quantizing the spin of the atom in the direction of emission of the photon $(\hat{\mathbf{k}})$, we get the following expression for the density matrix.

$$
\begin{aligned}
\rho_{00}^{0} & =1 \\
\rho_{M M}^{1} & =\frac{1}{3}\left\{+\frac{3}{2} M(\mathbf{P} \cdot \mathbf{k})\right\}
\end{aligned}
$$

and substituting in the angular distribution (3.8)

$I^{\left(F, F^{\prime}\right)}(\theta)=\frac{W\left(F, F^{\prime}\right)}{4 \pi}\left\{1+\frac{3}{2\left(F^{\prime}+1\right)} a\left(F, F^{\prime}\right) \mathbf{P} \cdot \mathbf{k}\right\}$

where $W\left(F, F^{\prime}\right)=\sum_{\varepsilon=+,-} W_{\varepsilon}\left(F, F^{\prime}\right)$ and $a\left(F, F^{\prime}\right)$ is the photon polarization defined in (3.6).

Finally, the angular asymmetry, defined as:

$\mathscr{A}\left(F, F^{\prime}\right)=\frac{I^{\left(F, F^{\prime}\right)}(\theta)-I^{\left(F, F^{\prime}\right)}(\pi-\theta)}{I^{\left(F, F^{\prime}\right)}(\theta)+I^{\left(F, F^{\prime}\right)}(\pi-\theta)}$

is given by:

$\mathscr{A}\left(F, F^{\prime}\right)=\frac{3}{F^{\prime}+1} \delta_{F, 1} a\left(F, F^{\prime}\right)|\mathbf{P}| \cos \theta$

where $\theta$ is the angle between the initial polarization of the atom and the direction of the emitted photons.

Observables $(3.6,3.12)$ are based on the measurement of the dipole magnetic radiation, they give a direct evaluation of the mixing parameter $\eta$ and hence they make contact with the weak neutral current and the parameters of the theory.

Let us finish this section with two comments.

The parameter $\eta(0, J)$ is not involved in the angular asymmetry. Being the initial spin of the atom equal to zero, it is an isotropic system (see (3.7)) and we cannot consider the observable described above. We can however relate the final polarization of the atom with this parameter. Since:

$\mathbf{P}_{f} \cdot \hat{\mathbf{k}}=\frac{\operatorname{Tr}\left(\rho^{F^{\prime}} \mathbf{F}^{\prime} \cdot \hat{\mathbf{k}}\right)}{\operatorname{Tr} \rho^{F^{\prime}}}=\frac{\left(\Delta E_{F F^{\prime}}\right)^{2}}{4 \pi} \frac{\operatorname{Tr}\left(A \rho^{0} A^{+} \mathbf{F}^{\prime} \cdot \hat{\mathbf{k}}\right)}{I^{(0,1)}(\theta)}$ we have:

$\mathbf{P}_{f} \cdot \hat{\mathbf{k}}=-a(0,1)$

and hence the relation with $\eta(0, J)$.

Finally, we can deduce the case with a zero spin mucleus $(I=0$ in (3.1)) by just considering the mixing with $2 S_{1 / 2}$ and the part of the weak Hamiltonian independent on the nuclear spin.

\section{Neutral weak currents and the muon-nucleus interaction}

At low energies, the neutral current interactions can be analysed from the point of view of an effective theory with current $x$ current couplings between the different fermions. With the only assumptions of vector and axial-vector structure for the currents, flavour conserving and universality of lepton couplings, it is possible to obtain an effective Lagrangian [6] which, in terms of thirteen parameters (for two generations of fermions), describes all the phenomenology of electroweak neutral current interactions.

In particular, we are interested in the lepton-quark sector, which in the Hung-Sakurai parametrization is described in terms of four independent coupling constants. Calling our attention to the spin and isospin dependence, they are: $\tilde{\alpha}$ (vector, isovector), $\widetilde{\beta}$ (axialvector, isovector), $\tilde{\gamma}$ (vector, isoscalar) and $\tilde{\delta}$ (axialvector, isoscalar).

In this section we will work out the relation between the coupling of quarks and leptons (through the coupling constants mentioned above) and the mixing parameter $\eta(F, J)$, thus finding the relation between the fundamental parameters of the theory and the experimental information described by the observables $(3.6,3.12)$.

The effective Hamiltonian which describes processes mediated by weak neutral currents is:

$H_{W}=\frac{G}{\sqrt{2}} J_{\mu}(x) J^{\mu}(x)$

where $G$ is the Fermi constant determined by muon decay and the current $J_{\mu}(x)$ contains the pieces vector and axial-vector for both the leptonic and hadronic current. Explicitly:

$J_{\mu}(x)=\sum_{\text {leptons }} J_{\mu}^{l}(x)+\sum_{\text {quarks }} J_{\mu}^{q}(x)$

where

$J_{\mu}^{l}(x)=\bar{l}(x) \gamma_{\mu}\left(v_{l}-a_{l} \gamma_{5}\right) l(x)$

$J_{\mu}^{q}(x)=\bar{q}(x) \gamma_{\mu}\left(v_{q}-a_{q} \gamma_{5}\right) q(x)$

and $v_{i}\left(a_{i}\right)$ are the vector (axial-vector) couplings, for leptons and quarks.

In the framework of the electroweak standard model $S U(2) \otimes U(1)$, these couplings are completely determined in terms of one parameter, the so-called Weinberg angle $\left(\theta_{W}\right)$. The explicit relations are, for 
two generations of fermions:

$$
\begin{aligned}
& v_{u}=\frac{1}{2}-\frac{4}{3} \sin ^{2} \theta_{W} \\
& a_{u}=\frac{1}{2} \\
& v_{d}=-\frac{1}{2}+\frac{2}{3} \sin ^{2} \theta_{W} \\
& a_{d}=-\frac{1}{2}
\end{aligned}
$$

and, assuming universality in the leptonic sector:

$v_{l}=-\frac{1}{2}+2 \sin ^{2} \theta_{W}$

$a_{l}=-\frac{1}{2}$.

In more general models, the coupling constants depend on the coupling of fermions to the different bosons and in general, more than one parameter is needed to completely determine them. For a clear treatment of this subject, see [13].

In the remainder of the section we will describe the relation between the coupling constants among quarks and leptons and the effective Hamiltonian describing the weak interaction between nucleus and muons. So we will establish the necessary bridge between the observables presented in Sect. 2 and the fundamental parameters of the theory.

At low energies the quarks act coherently with regard to the weak interaction. All the information about the strong interactions of quarks inside the nucleon can be included in the Lorentz invariant form factors. However, in this case, the momentum transfer is very small as compared to any other mass scale of the problem, hence one can use the approximations for the nucleonic currents:

$$
\begin{aligned}
& \left\langle\mathbf{p}_{1}\left|J_{\mu}^{q}(V)\right| \mathbf{p}_{2}\right\rangle=f_{1}(0) v_{q} \bar{u}\left(\mathbf{p}_{1}\right) \gamma_{\mu} u\left(\mathbf{p}_{2}\right) \\
& \left\langle\mathbf{p}_{1}\left|J_{\mu}^{q}(A)\right| \mathbf{p}_{2}\right\rangle=g_{1}(0) a_{q} \bar{u}\left(\mathbf{p}_{1}\right) \gamma_{\mu} \gamma_{5} u\left(\mathbf{p}_{2}\right)
\end{aligned}
$$

where $J_{\mu}^{q}(V)\left(J_{\mu}^{q}(A)\right)$ stands for the vector (axialvector) piece of the currents (4.3), $f_{1}$ and $g_{1}$ are the corresponding form factors at $q^{2}=0$. The hypothesis of conserved vector current relates the coupling $f_{1}$ to the electromagnetic form factor thus $f_{1}(0)=1$, on the other hand the hypothesis of partially conserved axial current relates $g_{1}$ to $f_{1}$ [8] for the isovector current:

$\frac{g_{1}(0)}{f_{1}(0)}=1.25 \pm .01$

$\left(g_{1}(0)\right.$ is usually referred to as the axial coupling $g_{A}$ of the nucleon). Similarly, with $g_{A}^{0}$ the renormalization of the axial isoscalar coupling constant [18] for the nucleon is taken into account, with 0.75 as the canonical prediction.

The parity violating piece of the effective Hamiltonian (2.2) is then $\left(H=H_{1}+H_{2}\right)$ :

$$
\begin{aligned}
H_{1}= & \frac{G}{2 \sqrt{2}}\left\{C_{1 p} \bar{\psi}_{p}(x) \gamma_{v} \psi_{p}(x)+C_{1 n} \bar{\psi}_{n}(x) \gamma_{v} \psi_{n}(x)\right\} \\
& \cdot \bar{\psi}_{\mu}(x) \gamma^{v} \gamma^{5} \psi_{\mu}(x)
\end{aligned}
$$

$$
\begin{aligned}
H_{2}= & \frac{G}{2 \sqrt{2}}\left\{C_{2 p} \bar{\psi}_{p}(x) \gamma_{v} \gamma^{5} \psi_{p}(x)+C_{2 n} \bar{\psi}_{n}(x) \gamma_{v} \gamma^{5} \psi_{n}(x)\right\} \\
& \bar{\psi}_{\mu}(x) \gamma^{\nu} \psi_{\mu}(x)
\end{aligned}
$$

written in terms of the fields and the coupling constants of the nucleons. Both Hamiltonians have the structure vector $\times$ axial-vector current, suitable for parity violating transitions. For convenience we have split the effective Hamiltonian into two pieces with regard to the dependence on the nuclear spin (see below).

The coupling constants in (4.8) are products of couplings of quarks and muons since they represent the effective coupling by the exchange of a massive boson. The explicit form can be readily found from $(4.4,4.5)$ and the quark content of nucleons.

Finally, the effective potential describing the muon and nucleus weak interaction is obtained considering a non-relativistic description of the motion of the nucleons inside the nucleus and using the impulse approximation, i.e. representing the interaction between the nucleus and muons by the one with single nucleons.

In the Hamiltonian (4.8) both hypothesis amount for the substitutions $\gamma^{0} \rightarrow 1, \gamma \sim \gamma^{0} \gamma^{5} \sim 0$ and $\gamma \gamma^{5} \sim \sigma$ in the nucleonic current and, as a consequence:

$$
\begin{aligned}
& \bar{N}(x) \gamma^{0} N(x) \rightarrow \sum_{i=1}^{A} \delta\left(\mathbf{x}-\mathbf{x}_{i}\right) \\
& \bar{N}(x) \gamma^{0} \tau_{3} N(x) \rightarrow \sum_{i=1}^{A} \tau_{3}^{(i)} \delta\left(\mathbf{x}-\mathbf{x}_{i}\right) \\
& \bar{N}(x) \gamma \gamma^{5} N(x) \rightarrow \sum_{i=1}^{A} \boldsymbol{\sigma}_{i} \delta\left(\mathbf{x}-\mathbf{x}_{i}\right) \\
& \bar{N}(x) \gamma \gamma^{5} \tau_{3} N(x) \rightarrow \sum_{i=1}^{A} \boldsymbol{\sigma}_{i} \tau_{3}^{(i)} \delta\left(\mathbf{x}-\mathbf{x}_{i}\right) .
\end{aligned}
$$

In these equations $N(x)$ represents a proton-neutron isospin doublet, $\tau_{3}^{(i)}$ is the third-component of the Pauli matrices and $\mathbf{x}_{i}$ stands for the nucleon position in the nucleus. The right hand side must be understood sandwiched between nuclear states. The remaining components of the effective Hamiltonian are zero in this limit.

Going back to (4.8) the effective potential reads (using the Hung-Sakurai parametrization for the coupling constants):

$$
\begin{aligned}
& H_{1}=-\frac{G}{2 \sqrt{2}}\left\{\sum_{i=1}^{A} \delta\left(\mathbf{x}-\mathbf{x}_{i}\right)\left[3 \tilde{\gamma}+\tau_{3}^{(i)} \tilde{a}\right]\right\} \gamma^{0} \gamma^{5} \\
& H_{2}=\frac{G}{2 \sqrt{2}}\left\{\sum_{i=1}^{A} \delta\left(\mathbf{x}-\mathbf{x}_{i}\right)\left[3 g_{A}^{0} \tilde{\delta}+g_{A} \tilde{\beta} \tau_{3}^{(i)}\right] \boldsymbol{\sigma}_{i}\right\} \gamma
\end{aligned}
$$

where $\gamma^{0} \gamma^{5}$ and $\gamma$ act on the muon wave functions.

The coupling constants are defined in terms of the quark and muon couplings $(4.4,4.5)$ as:

$\begin{array}{ll}\tilde{\alpha}=2 a_{l}\left(v_{u}-v_{d}\right) & \tilde{\beta}=2 v_{l}\left(a_{u}-a_{d}\right) \\ \tilde{\gamma}=2 a_{l}\left(v_{u}+v_{d}\right) & \tilde{\delta}=2 v_{l}\left(a_{u}+a_{d}\right) .\end{array}$ 
The decomposition of the weak Hamiltonian given in (4.11) has been done with regard to the nuclear spin. The isoscalar piece $\left(H_{1}\right)$ will give the general contribution, it depends coherently on the total number of quarks in the nucleus being the dominant term in heavy atoms as well as the only contributing term in atoms with spinless nucleus. As it was mentioned in the introduction it provides information about a definite combination of $\tilde{\alpha}$ and $\tilde{\gamma}$. On the other hand, the vectorial piece $\mathrm{H}_{2}$ depends on the nuclear spin, namely its contribution is given by the nucleons outside closed shells. Hence, at the present accuracy of the experiments, it is negligible in heavy atoms. However, in light atoms with non-zero spin of the nucleus, its contribution can be as important as the one coming from $H_{1}$.

The second piece presents the novelty of being sensitive to the coupling constants $\widetilde{\beta}$ and $\widetilde{\delta}$ that have not been measured in the present experiments with heavy atoms. Henceforth, since light atoms with spin are sensitive to these coupling constants they provide a suitable system in looking for information about them.

It is worth mentioning that in the framework of the standard model these couplings are very small, namely $\widetilde{\delta}=0, \widetilde{\beta}=-1+4 \sin ^{2} \theta_{W}$. This, however, provides the prospect of being sensitive to Physics that is not described by the standard model $[5,17]$.

\section{Results and conclusions}

With the help of the formalism we developed in Sects. 3 and 4 we can now find the relation between the observables $(3.6,3.12)$ and the coupling constants $\tilde{\alpha}, \widetilde{\beta}, \tilde{\gamma}, \tilde{\delta}$. We will present here the form of the mixing parameter and give the results for the expected photon polarization and angular asymmetry.

As we commented before, only $2 P_{J}$ states give appreciable mixing. Thus, using $(3.1,3.2)$, we have explicitly:

$$
\begin{aligned}
& \eta(F, J)=\sum_{m_{1} m_{2}}\left(\frac{1}{2} \frac{1}{2} F \mid m_{1} M-m_{1}\right)\left(J \frac{1}{2} F \mid m_{2} M-m_{2}\right) \\
& \frac{\left\langle 2 P_{J m_{1}}\left|\left\langle\frac{1}{2} M-m_{1}\left|H_{1}+H_{2}\right| \frac{1}{2} M-m_{2}\right\rangle\right| 2 S_{1 / 2} m_{2}\right\rangle}{E\left(F, 2 S_{1 / 2}\right)-E\left(F, 2 P_{J}\right)} .
\end{aligned}
$$

In light atoms it is appropriate to ignore the finite size of the nucleus. Then, in (4.11) we can put $\mathbf{x}_{i}=0$ choosing the origin of coordinates on the nucleus. The calculation of the matrix elements in (5.1) is then straightforward. The results reads:

$$
\begin{aligned}
\eta\left(F, \frac{1}{2}\right)= & -i \frac{G}{\sqrt{2}} \frac{\sqrt{3}}{64 \pi} \frac{(\alpha z)^{4} m_{\mu}^{3}}{\Delta E}\{3 \tilde{\gamma}(Z+N)+\tilde{\alpha}(Z-N) \\
& -\sqrt{3}(-)^{1-F} W\left(\frac{1}{2} \frac{1}{2} \frac{1}{2} \frac{1}{2} \mid 1 F\right) \\
& \cdot\left\langle\frac{1}{2}\left\|\sum_{i=1}^{A}\left[3 g_{A}^{0} \tilde{\delta}+g_{A} \tilde{\beta} \tau_{3}^{(i)}\right] \sigma_{i}\right\| \frac{1}{2}\right\rangle
\end{aligned}
$$

$\eta\left(F, \frac{3}{2}\right)=0$

where $\Delta E=E\left(F, 2 S_{1 / 2}\right)-E\left(F, 2 P_{1 / 2}\right)$, i.e. the difference between the hyperfine admixed states, $W$ is the Racah coefficient and the reduced matrix element for the isovector current can be related to the GamowTeller operator appearing in $\beta$-decay [14]. The matrix element of the axial isoscalar current has to be calculated or extracted [18] from the isoscalar magnetic moment.

The vanishing of the mixing parameter (5.3) comes from the vanishing of the $2 P_{3 / 2}$ muonic wave function at the origin. It can be understood by performing a non-relativistic reduction of the effective Hamiltonian in (4.11). The scalar piece does not contribute since the matrix element for $\eta\left(F, \frac{3}{2}\right)$ entails change in the muonic angular momentum. On the other hand the operator $\gamma$ gives two components, the spin current described by the operator $\mathbf{p} \times \boldsymbol{\sigma}$ and the convection current given by the operator $\mathbf{p}$. As it happens they cancel each other in (5.3). Consideration of the finite size of the nucleus gives a non-vanishing contribution to (5.3), however, considering that typically ( $R_{\text {nucleus }} /$ $\left.a_{\mu}\right) \sim 10^{-2}$ in light atoms, we can in general neglect this effect.

Before going on let us, once again, stress the main features of the mixing parameter. On one hand, the factor $m_{\mu}^{3}$ gives an enhancement of the order of $10^{7}$ when compared to electronic atoms. On the other hand, the scalar piece is dominant in heavy atoms since it depends on the coherent contributions of the nucleons and, to the present, the spin dependent part has not been measured. The same comment is also valid for a spinless nucleus whose only contribution is given by this scalar piece. So the new information given by light atoms is contained in the spin dependent piece of the mixing parameter.

In the following we calculate the mixing parameter (5.2) for $\mu-{ }^{3} \mathrm{He}$ and $\mu-{ }^{4} \mathrm{He}$ in the standard theory, emphasizing the contribution from the scalar and vectorial components. We take $\sin ^{2} \theta_{W}=0.226 \pm 0.004$ [16] and the difference between the energy levels given in [15]. The results for the mixing parameter and the photon polarization are quoted on Table 1 . As we mentioned before, the results for $\mu-{ }^{4} \mathrm{He}$ are obtained from the expression (5.2) just considering the spin independent part. For the sake of comparison we have split the results in the contribution coming from the

Table 1. The mixing parameter and photon polarization for muonic atoms $\mu-{ }^{3} \mathrm{He}$ and $\mu-{ }^{4}$ He. The first (second) term corresponds to the scalar (vectorial) contribution from the effective Hamiltonian.

\begin{tabular}{lll}
\hline & $\mu-{ }^{3} \mathrm{He}$ & $\mu-{ }^{4} \mathrm{He}$ \\
\hline $\operatorname{Im} \eta\left(0, \frac{1}{2}\right)$ & $(2.7-0.5) \times 10^{-9}$ & $5.0 \times 10^{-9}$ \\
$\operatorname{Im} \eta\left(1, \frac{1}{2}\right)$ & $(2.5-0.2) \times 10^{-9}$ & \\
$a\left(0, F^{\prime}\right)$ & $(-1+0.2) \times 10^{-2}$ & $-2 \times 10^{-2}$ \\
$a\left(1, F^{\prime}\right)$ & $(-1+0.07) \times 10^{-2}$ & \\
\hline
\end{tabular}


scalar and vectorial pieces (see Table 1), the second one being of the order of $10-20 \%$ of the first one.

Finally, these results give a forward-backward angular asymmetry (3.12) of the order of a few percent for a completely polarized atom. Notice that the net effect of the nuclear spin is the decreasing of the signal as the associated term interferes destructively with the dominant scalar term. Similar calculations for atoms other than $\mathrm{He}$ can be carried out along the lines presented here.

To summarize, we have reviewed the effect of the weak neutral currents in light muonic atoms. As we have discussed, these systems are suitable to study the spin dependent piece of the effective weak Hamiltonian and hence the weak coupling constants $\widetilde{\beta}$ and $\tilde{\delta}$. The enhancement factors were established to be: the overlapping between the muonic and nuclear probability, the nearly degenerate states of opposite parity and the selection of transitions whose parity allowed amplitude is suppressed. These facts give the $2 S-1 S$ transition as a good candidate to the search of these coupling constants through the neutral current effect in light muonic atoms.

In order to consider the effect of the nuclear spin, we have calculated the values of the photon polarization (3.6) and the angular asymmetry (3.12) for ${ }^{3} \mathrm{He}$ and ${ }^{4} \mathrm{He}$. The theoretical result for the asymmetry is appreciable, of the order of a few percent. In the standard theory the contribution from the nuclear spin dependent piece was found to be of the order of $10-20 \%$ of the dominant scalar one. In general, this piece depend on physics beyond the standard model through the axial isoscalar neutral current coupling.

Acknowledgements. This work has been supported in part by CICYT, under grant AE-0021. Two of us (J.B. and J.V.) are indebted to Ministerio de Educación y Ciencia and to C.S.I.C., respectively for the research fellowships. J.B. wishes to thank the Rutherford Laboratory for hospitality.

\section{References}

1. M.A. Bouchiat, C. Bouchiat: Phys. Lett. 48B (1974) 111; M.A. Bouchiat, L. Pottier: Atomic physics 9, R.S. Van Dyke Jr. E.N. Forston (eds.) Singapore: World Scientific 1984

2. P. Langacker: Proceedings of 1985 International Symposium on Lepton Photon Int. at High Energies, Kyoto, 1985. M. Konuma, K. Takahashi (eds.) Kyoto: Nissha 1985

3. M.C. Noecker, B.P. Masterton, C.E. Wieman: Phys. Rev. Lett. 61 (1988) 310; S.L. Gilbert, M.C. Noecker, R.W. Watts, C.E. Wieman: Phys. Rev. Lett. 55 (1985) 2680; M.A. Bouchiat, J. Guéna, L. Hunter, L. Pottier: Phys. Lett. 134B (1984) 463; C.A. Piketty: Parity violation in atoms. Proceedings of the International Symposium. Heidelberg, H.V. Klapdor (ed.) Berlin, Heidelberg, New York: Springer 1986; P. Bucksbaum, E. Commins, L. Hunter: Phys. Rev. Lett. 46 (1981) 640

4. R.R. Lewis, W.L. Williams: Phys. Lett. 59B (1975) 70; E.A. Hinds V.W. Hughes: Phys. Lett. 67B (1977) 487; E.A. Hinds: Phys. Rev. Lett. 44 (1980) 374; E.G. Adelberger et al.: Nucl. Instrum Methods 179 (1981) 181; L.P. Levy W.L. Williams: Phys. Rev. Lett. 48 (1982) 607

5. J. Bordes: Phys. Lett. 190B (1987) 97

6. P.Q. Hung, J. Sakurai: Ann. Rev. Nucl. Part. Sci. 31 (1981) 375 J. Bernabéu, T.E.O. Ericson, C. Jarlskog: Phys. Lett. 50B (1974) 467; G. Feinberg, M.T. Chen: Phys. Rev. D10 (1974) 190

8. C. Jarlskog: In Proc, of the 1974 CERN School of Physics (1974)

9. L.M. Simons: Possibilities to measure electroweak effects in muonic atoms. Proceedings of the International Symposium, Heidelberg. H.V. Klapdor (ed.). Berlin, Heidelberg, New York: Springer 1986; R. Bacher et al:: Paul Scherrer Institute Preprint PR-88-14

10. P. Blüm et al:: Proposals SIN R-81-02, 1 (1981)

11. J. Missimer, L. Simons: Phys. Rep. 118 (1985) 179

12. H.A. Bethe, E.E. Salpeter: Quantum mechanics of one and two electrons atoms. Berlin, Göttingen, Heidelberg: Springer 1957

13. F. del Aguila, M. Quirós, F. Zwirner: Nucl. Phys. B284 (1987) 530

14. J. Bernabéu: Astron. Astroph. 47 (1976) 375

15. C. Campani: Lett. Nuovo Cimento 4 (1970) 982

16. Review of Particle Properties: Phys. Lett. 170B (1986)

17. R.N. Cahn, G.L. Kane: Phys. Lett. 71B (1977) 348

18. J. Bernabéu, R.A. Eramzhyan: Phys. Lett. 80B (1979) 396 